\title{
Effects of autologous platelet-rich plasma on skin healing in dogs
}

\author{
S.M. Hussein \\ Department of Animals Production, College of Agriculture, Sumer University, Iraq \\ Email: moh07709879860@gmail.com
}

(Received March 7, 2018; Accepted May 16, 2018)

\begin{abstract}
This study aimed to evaluate the benefits of using platelet- rich plasma (PRP) on wound healing. The study performed on ten adult male dogs, aged 2-3 years and their weight were 18-25 kg. Under the effect of deep sedation and local subcutaneous anesthesia at the site of wound, four of $(2 \times 2) \mathrm{cm}$ of full-thickness square cutaneous wounds were induced, two on each side of the lateral thoracic region of each animal. A pair of left wounds was treated by injection with $5 \mathrm{~mL}$ of autonomous PRP (treatment group), $2 \mathrm{~mm}$ lateral to the wound edges and in the wound center., immediately after wounding while, right wounds, were injected with a single dose of $5 \mathrm{ml}$ of sterile saline (control group) by using the same procedure as in treatment group. The clinical and histopathological evaluation of each group for five weeks, histological biopsies were taken in the days $(3,7,14,21,28$ and 35) of the experiment. The clinical evaluation of PRP treated wounds showed that the wound healing process $(\%$ contraction, $\%$ re-epithelization and \% total wound healing) were significantly more $(\mathrm{P}<0.05)$ than the control wounds during the five weeks study. The histopathological results revealed that PRP treated wounds have enhanced cellularity, increased vascularity, with increased amount of granulation tissue accompanied by increase numbers of skin appendages suggesting enhanced cutaneous regeneration, than those in untreated wounds. Depending on the clinical and histopathological findings, this study confirms that local implantation of PRP leads to acceleration and improvement cutaneous wound healing.
\end{abstract}

Keywords: Dogs, Wound healing, Platelet- rich plasma, Growth factors, injery, skin Available online at http://www.vetmedmosul.com

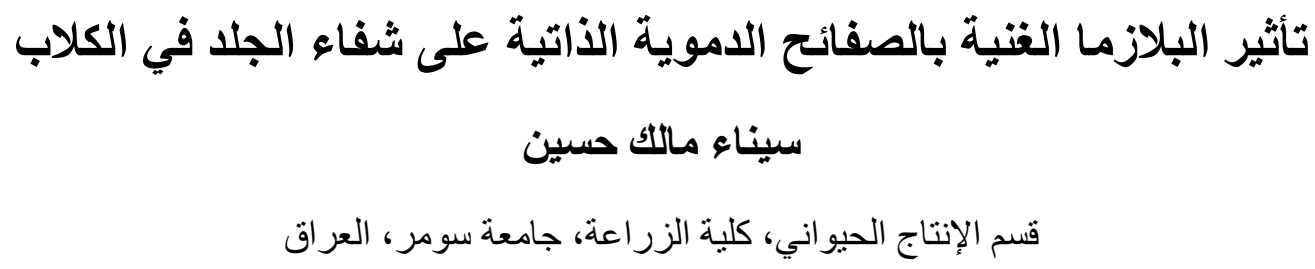

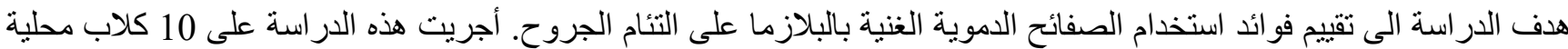

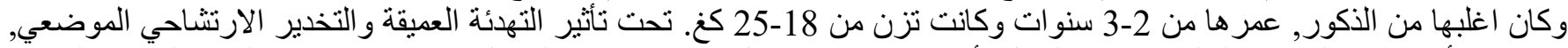

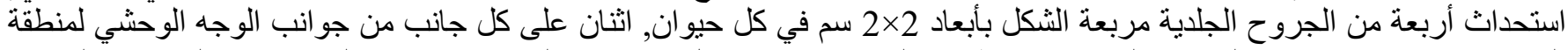

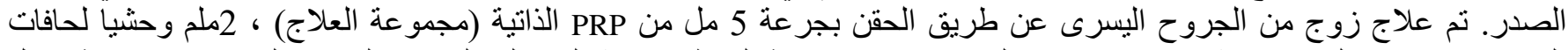

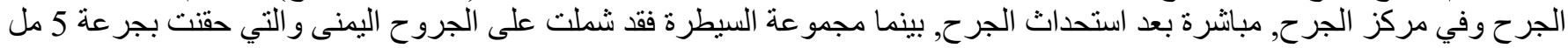

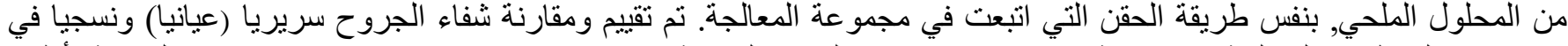

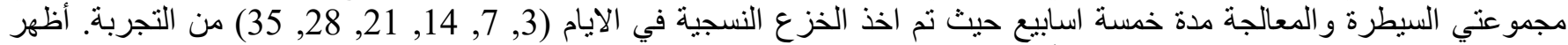

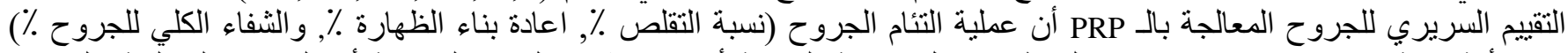

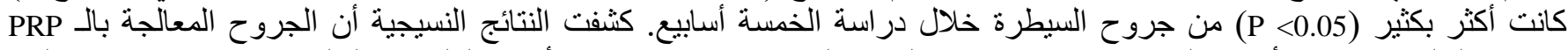

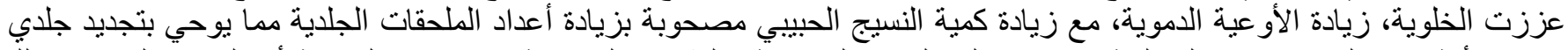

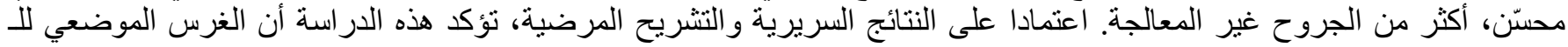

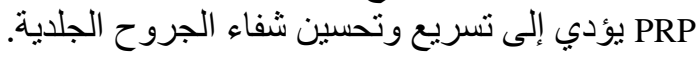




\section{Introduction}

Wound repairing is complexes multifactorial processthose outcomes in the constriction and conclusion of the imperfection and rebuilding of a useful boundary. This process occurs as a sequence of events including hemostasis, inflammatory cell infiltration, tissue regrowth, and remodelin $(1,2)$. Wound healing biologists aim to understand how a wound healing procedure can be induced to repair the damaged tissues faster and more efficiently $(3,4)$. Enhancement of dermal and epidermal regeneration is an extremely important goal for the treatment of many different types of wounds (5). Wound healing is regulated by several cell types and by a cascade of peptides such as cytokines or growth factors. Following injury, growth factors secretion by platelets and macrophages are induced and inflammatory process which is needed for healing is initiated (6). Another region of tissue designing including bioactive atom based medicines has at present increased much consideration. A few examinations have demonstrated that cytokine treatment may quicken mending of tissues and particularly advance the repair of disabled injuries in the assortment of creatures. (7-9), unfortunately, these purified bioactive agents has fallen short of expectations in clinical studies, as it is currently clear that no single exogenous agent can effectively mediate all aspects of wound healing process (10), may be due to the fact that cytokines work in concert, both temporally and spatially. (12) So, the dynamic idea of wound repair process substantiates the requirement for cytokine blend treatments (13). In an effort to provide this combination treatment, investigators have turned to Platelet-rich plasma (PRP), which is a rich source of different cytokines essential for natural healing process. It is released from platelet $\alpha$ granules at sites of tissue injury, $(14,15)$. These include catecholamines, serotonin, adenosine triphosphate (ATP), albumin, fibrinogen, osteonectin, osteocalcin and calcium ions. Various clotting factors and locally active growth factors, such as platelet-derived growth factor (PDGF), transforming growth factor- $\alpha$ (TGF- $\alpha$ ), transforming growth factor- $\beta$ (TGF- $\beta$ ), insulin-like growth factor (IGF), fibroblast growth factor (FGF), vascular endothelial growth factor (VEGF) and epidermal growth factor (EGF) also initiate wound healing. (16-18). Platelets also secrete fibrin, fibronectin, and vitronectin, which provide a matrix for connective tissue and act as adhesion molecules for epithelial migration $(19,20)$.

The later stimulates angiogenesis, promoting vascular in growth and fibroblasts proliferation. In addition, it acts as hemostatic effect by forming a fibrin clot. Also application of PRP enhances wound healing in both soft and hard tissue. PRP is $100 \%$ biocompatible and safe. It poses absolutely no infectious risk to the patient because it is made from the patient's own plasma $(21,22)$. A literature to assess the current clinical experience and the possible effects of (PRP) on wound-healing yields recorded by few reports (23), thus the present study was designed to detect the role of PRP in healing of experimentally wounded skin in dogs $(24,25)$.

\section{Materials and methods}

\section{Preparation of platelet-rich plasma}

Blood sample $20 \mathrm{~mL}$ of autologous blood withdrawn from each dog was collected under a septic technique from the jugular vein of each dog via a 21 gauge needle and deposited in $3.2 \%$ sodium citrate tube with capacity for 10 $\mathrm{mL}$. Then placed in a centrifuge at $3200 \mathrm{rpm}$ for $15 \mathrm{~min}$. During centrifugation, blood is separated into three different fractions: platelet-poor plasma (superior layer), white blood cells (intermediate layer) and red blood cells (inferior layer) (Fig. 1). The first supernatant plasma fraction (50\%), adjacent to the buffy coat, was obtained under aseptic conditions in a laminar flow chamber. This fraction was centrifuged at $3200 \mathrm{rpm}$ for another 15 minutes in order to obtain two parts: the upper part which is platelet poor plasma PPP and the lower part was the PRP (25\%). Then the PRP was aspirated with another pipette and placed in a sterile tube and activated with calcium chloride (4.5 $\mathrm{mEg} / 5 \mathrm{~mL}$ ), using $50 \mu \mathrm{L} / \mathrm{mL}$ of PRP, to provide a gel matrix for a PRP to adhere to the site of injection (26). An average of $5 \mathrm{ml}$ of PRP was obtained from every $20 \mathrm{ml}$ of whole blood. A fraction of $1 \mathrm{ml}$ of each animal was analyzed for platelet count. On the day of PRP obtainment, the platelets counts in PRP were 200. 000 to 350.000 cells $/ \mu \mathrm{L}$.

\section{Experimental animals and management}

Ten grown-up male cross breed canines, (2-3) years old and weighing, around (18-25 kg), were utilized as a part of this examination. The mutts were housed in pet hotels, sustained by business delicate upkeep abstains from food once every day and water was offered not indispensable.

\section{Pre-operative considerations}

The dorsal surface of thoracic districts was sans cut hair and arranged aseptically for the injuring. Thirty minutes prior to wounding, Penicillin-Streptomycin was administered I.M., in a dose of $20000 \mathrm{IU} / \mathrm{kg}$ and $10 \mathrm{mg} / \mathrm{kg}$ B.W., respectively. The dogs were premedicated by intramuscular administration of $2 \%$ Xylazine hydrochloride $0.05 \mathrm{mg} / \mathrm{kg}$.; anesthesia was accomplished by linear subcutaneous infiltration of lidocain hydrochloride $2 \%$ at the intended incision sites at a dose rate of $1 \mathrm{~mL} / 1 \mathrm{~cm}^{3}$.

\section{Technique of wounding}

Four regular square full-thickness skin wounds $(2 \times 2$ $\mathrm{cm}$ ) were made at the dorsal thoracic sides of each animal 
(two injuries on each side), $10-15 \mathrm{~cm}$ separated (Fig. 2). The aggregate number of the injuries is (40 wounds). The creatures submitted to two equivalent gatherings, the control gathering and the treatment gatherings. All injuries were not sutured (opened injuries). The trea ted wound were injected with $5 \mathrm{~mL}$ of autonomous PRP (treatment group), $2 \mathrm{~mm}$ lateral to the wound edges and in the wound center, immediately after wounding while, right wounds, were injected with a single dose of $5 \mathrm{ml}$ of sterile saline (control group) by using the same procedure as in treatment group.

After completion of wounds treatment in both groups, the wounds were bandaged by use of a protective, nonpressure and non-adherent dressing. The bandages have been changed, two days intervals and wounds were gently cleaned with gauze sponges soaked in normal saline, without debridement of the wound bed.

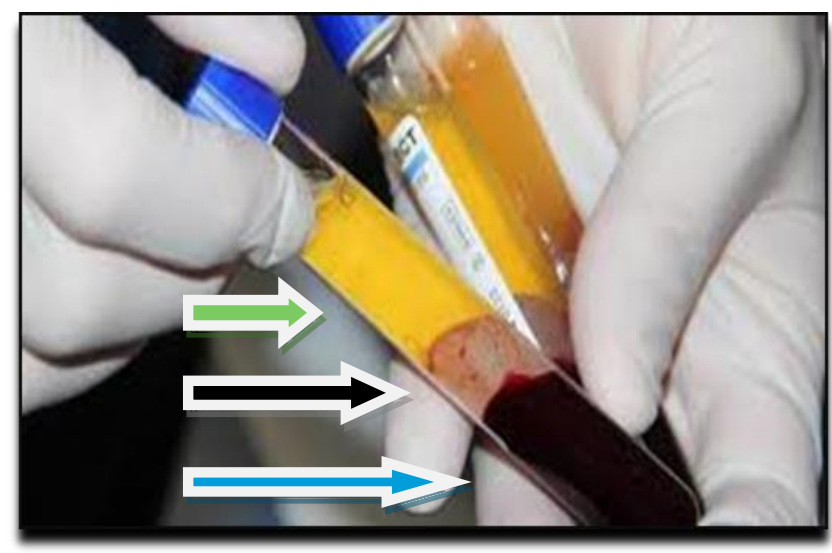

Fig. 1: Show's Preparation of PRP. Plasma of total blood (upper arrow), the leukocytes and platelets of total blood (middle arrow) and erythrocytes (lower arrow).

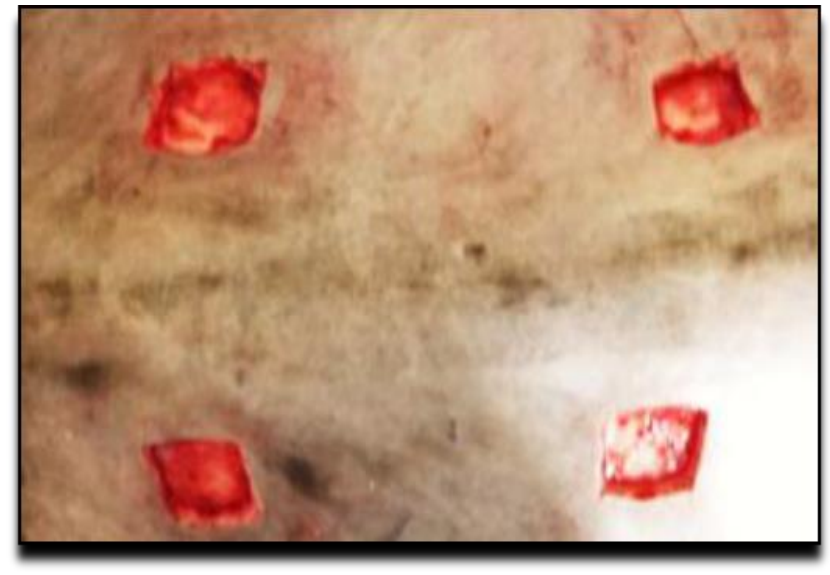

Fig. 2: Show's, the positions of squared wounds on lateral thoracic regions.

\section{Clinical and macroscopical evaluation}

A complete clinical examination was performed on all animals in days $(3,7,14,21,28$, and 35$)$ along the period of the study. Digital photographs were taken for all wounds after the area had been carefully shaved to visualize the wound margin. The scab of each wound was carefully removed for better visualization of the epithelialization and granulation tissue area by using saline. The percentages of epithelialization, wound contraction and total wound healing were calculated for each wound, depending on the parameters mentioned by (27).

\section{Percent of epithiliazation was calculated by:}

$\%$ Epithelialization $($ day $n)=$ Area of epithelium (day $n$ ) / Total wound area (day 0) x 100

\section{Percent of wound contraction, calculated by:}

Step 1: Total wound size on (day $n$ ) as $\%$ of original wound $=$ Total wound area $($ day $n) /$ Original wound area $($ day 0$) \mathrm{x}$ 100

Step 2: \% Wound contraction $($ day $n)=100-$ Total wound size on (day $n$ ) as $\%$ of original wound

\section{Percent of total wound healing, calculated by:}

Step 1: Open wound size day $n$ as $\%$ of original $=$ Open wound area (day $n$ ) / Original wound area (day 0) x 100 Step 2: \% Total wound healing $($ day $n)=100-$ Open wound size (day $n$ ) as \% of original wound

\section{Histopathologic evaluation}

At the day 3, 7, 14, 21, 28 and 35 after injuring, biopsies were taken from a similar corner of each twisted utilizing $0.9 \mathrm{~mm}$ biopsy punch for histopathological examination. The injury examples were settled in (10\%) unbiased support formalin arrangement. The tissue examples were prepared in a tissue processor, paraffin squares were frantic at (5-6) $\mu \mathrm{m}$ thick areas which were cut with a microtome and stained with Hematoxylin and Eosin dyes (28). Then stained slides were examined under light microscope.

\section{Statistical analysis}

The Statistical Analysis System- SAS (29) was used to effect of difference factors (treatment \& days) in study parameters (percentage). The least significant difference LSD test at the comparative between percentages in this study.

\section{Results}

\section{Clinical observations}

Amid a month consider, no any confusion, for example, wound disease or abundant granulation tissue arrangements in the injury locales of the two gatherings were taken note. Animals reflected normal appetite, urination and defecation 
within the first $24 \mathrm{~h}$ post-wounding. The after effects of key parameters, for example, temperature, respiratory and heart
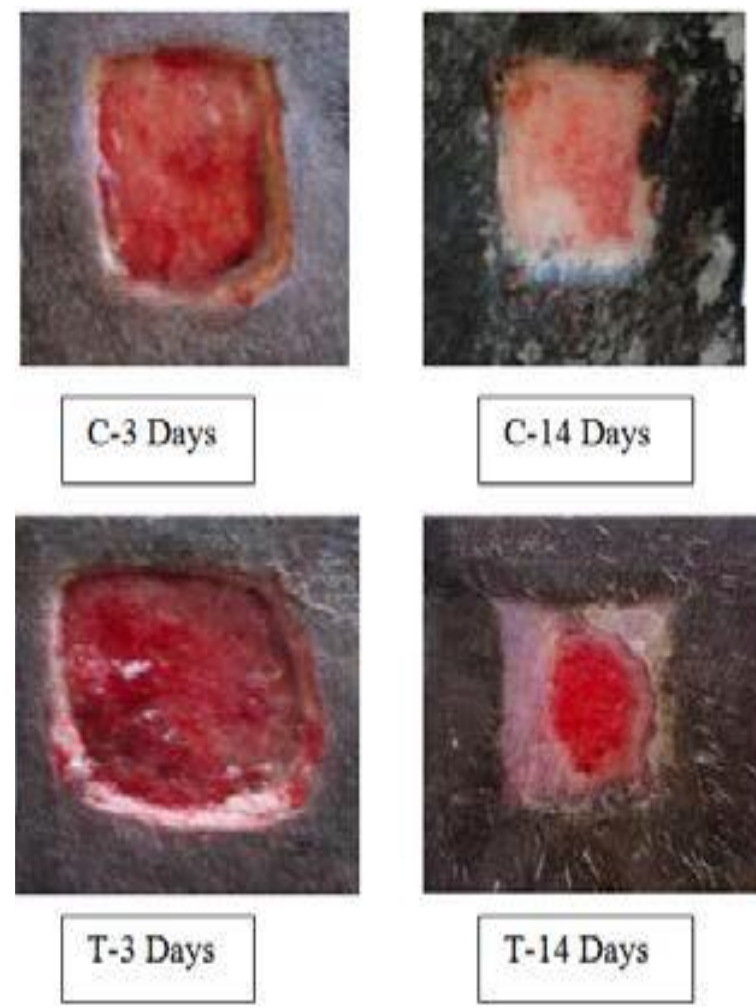

rates amid the main week post-injuring were inside the typical rang as specified in the most ward references.
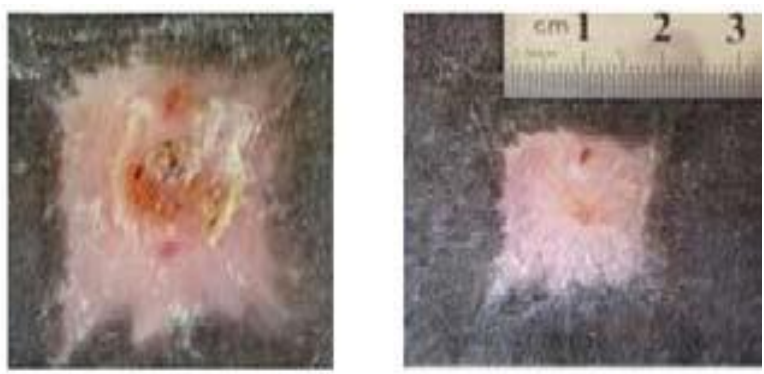

\section{C-21 Days}
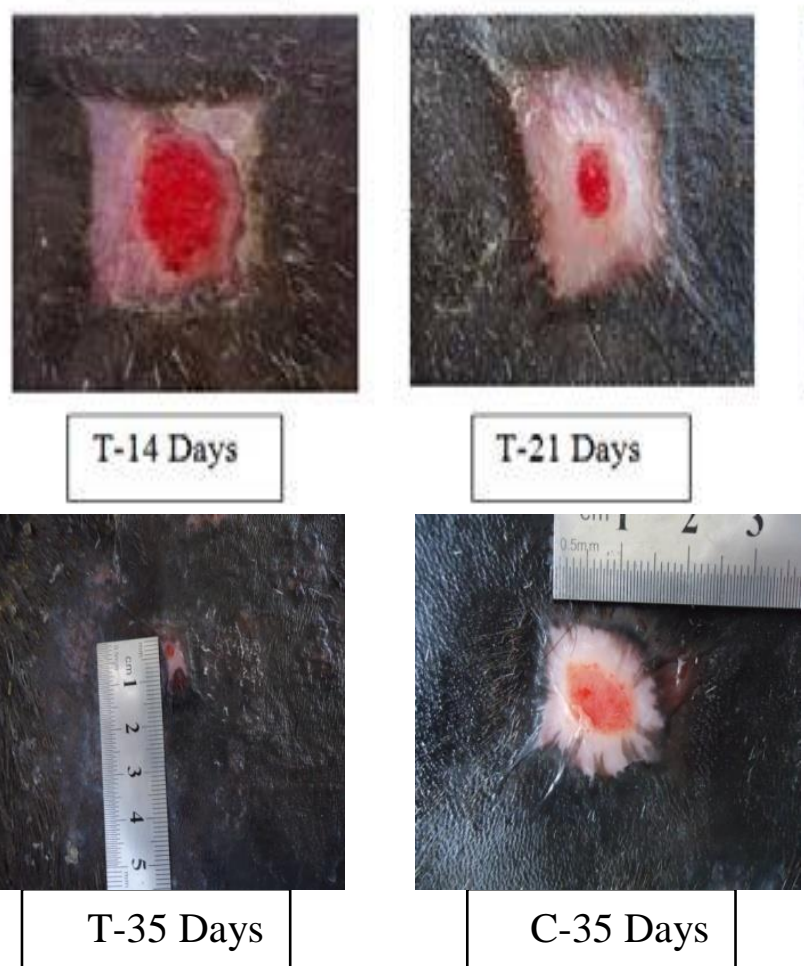

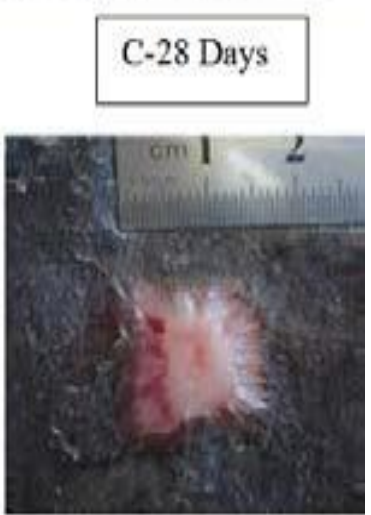

T-28 Days

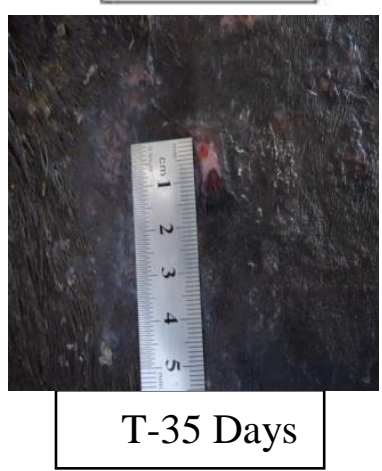

\section{T-14 Days}

T-35 Days

Fig. 3: Shows the gradual significant differences in total wound healing, pigmentation \& hair growth between control (C) and treatment $(\mathrm{T})$ wounds, according to the time.

\section{Wound morphometric analysis \\ Wound epithelialization}

The progress of epithelialization in treatment wounds was statistically significant $(\mathrm{P}<0.05)$ in comparing with control wounds, started from first week. The percent of epithelialization in control wounds was (31.1) and in treatment wounds was (72.10) at day 35 (Table 1).

\section{Wound contraction:}

There was significant differences $(\mathrm{P}<0.05)$ in speed of wound contraction between control and treatment groups started from first week. It was (76.66) and (84.44), respectively, at the end of the study (Table 2).

\section{Wound healing}

The rate of wound healing showed similar result as mentioned in wound contraction phenomena i.e., that the significant differences $(\mathrm{P}<0.05)$ between both group started from first week post-wounding. The highest rate was recorded in treatment group (89.44 at day 35) (Table3and Fig. 3). 
Table 1: Shows the second intension healing (effect of treatment and days) in re- epithelization (\%)

\begin{tabular}{cccc}
\hline Days & Control & Treatment & LSD value \\
\hline 3 & 0.0 & 0.0 & $0.00 \mathrm{NS}$ \\
7 & 0.0 & 0.0 & $0.00 \mathrm{NS}$ \\
14 & 13.3 & 13.3 & $0.00 \mathrm{NS}$ \\
21 & 24.4 & 42.2 & $6.733^{*}$ \\
28 & 3.11 & 5.00 & $2.743 \mathrm{NS}$ \\
35 & 31.1 & 63.30 & $8.503 *$ \\
LSD value & 31.1 & 72.10 & $9.893^{*}$ \\
& $12.477 *$ & $15.193 *$ & --- \\
\hline
\end{tabular}

* (P<0.05), NS: non-significant.

Table 2: Shows the second intension healing (effect of treatment and days) in Contraction (\%)

\begin{tabular}{cccc}
\hline Days & Control & Treatment & LSD value \\
\hline 3 & 0.0 & 0.0 & $0.00 \mathrm{NS}$ \\
7 & 0.0 & 0.0 & $0.00 \mathrm{NS}$ \\
14 & 31.44 & 50.00 & $4.155 \mathrm{NS}$ \\
21 & 40.00 & 53.33 & $4.209^{*}$ \\
28 & 60.00 & 67.77 & $5.215 *$ \\
35 & 68.88 & 75.56 & $5.839^{*}$ \\
LSD value & 76.66 & 84.44 & $6.316^{*}$ \\
& $15.328 *$ & $18.406 *$ & --- \\
\hline
\end{tabular}

* $(\mathrm{P}<0.05)$, NS: non-significant.

Table 3: Shows the second intension healing (effect of treatment and days) in total healing (\%)

\begin{tabular}{cccc}
\hline Days & Control & Treatment & LSD value \\
\hline 3 & 0.0 & 0.0 & $0.00 \mathrm{NS}$ \\
7 & 0.0 & 0.0 & $0.00 \mathrm{NS}$ \\
14 & 32.77 & 51.77 & $7.319^{*}$ \\
21 & 42.44 & 56.44 & $7.277 *$ \\
28 & 45.55 & 71.55 & $7.946 *$ \\
35 & 55.55 & 89.11 & $9.025 *$ \\
LSD value & 64.44 & 89.44 & $8.163 *$ \\
& $14.188 *$ & $18.452 *$ & --- \\
\hline
\end{tabular}

* $(\mathrm{P}<0.05), \mathrm{NS}$ : non-significant.

\section{Histopathological evaluation}

The histopathological examination of tissue biopsies from the wound peripheries and beds revealed that the main differences between treatment and control groups were started on day $7^{\text {th }}$ post-wounding. The histopathological sections of control group were showed necrotic debris with RBCs (hemorrhage) infiltration, fibrin network and small amount of granulation tissue (Fig.4). In addition, Fibrin network deposition with neutrophils infiltration and large number of fibroblasts were seen. While, in the treatment group, the wounds showed more advanced re-epithelization and to better angiogenesis, proliferation of fibroblasts, deposition of ECM and decrease of macrophages. No crust was observed on wound area. Therefore, the sections of treatment group, appeared that there is extensive granulation tissue extended and replaced most of the incision area with macrophage (Fig.5).

On day $14^{\text {th }}$ post-wounding, the histopathological observation in AD-MSCs treated wounds appeared that the lesion characterized by the presence of dens mature granulation tissue in the incision area with few number of fibroblast, as well as, thick epidermal area with rete ridge over the mature granulation tissue, numerous hair follicles and sebaceous glands were reformed (Fig.6). While, the sections of control group appeared the presence of few fibroblasts with few macrophages (Fig.7).

The sections of the control group on day 21postwounding, , the histopathological changes appeared the presence of thick epidermal layer over the granulation tissue with short rete ridge (Fig.8). Comparison to the treatment group on day 21post-wounding, appeared the presence of granulation tissue which infiltrated by mesenchymal cells characterized by keratinocyte and newly formed blood vessels variable in size that replaced the granulation tissue (Fig. 9). On day 2821post-wounding, the histopathological changes appeared the presence of epidermis layer cover the mature granulation tissue (Fig. 10); the histopathological sections of control group were showedMelanin pigment in the basal layer of epidermis cover the granulation tissue (Fig. 11). In another section, reformation of hair follicles and sebaceous glands (orang arrow) in the incisional site (Fig. 12)

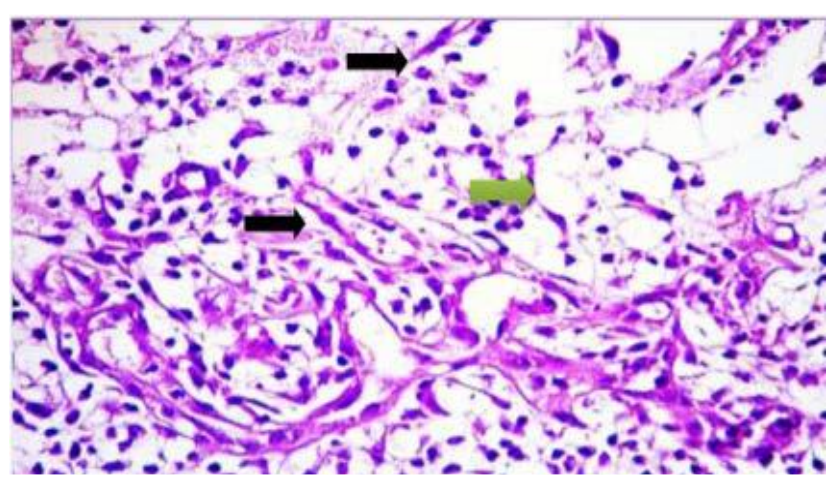

Fig. 4: Histopathologic section of wound related to control group, day $7^{\text {th }}$ post-wounding, show's neutrophils infiltration (green arrow) and fibrine net work formation (black arrows) (H\&E; X40). 


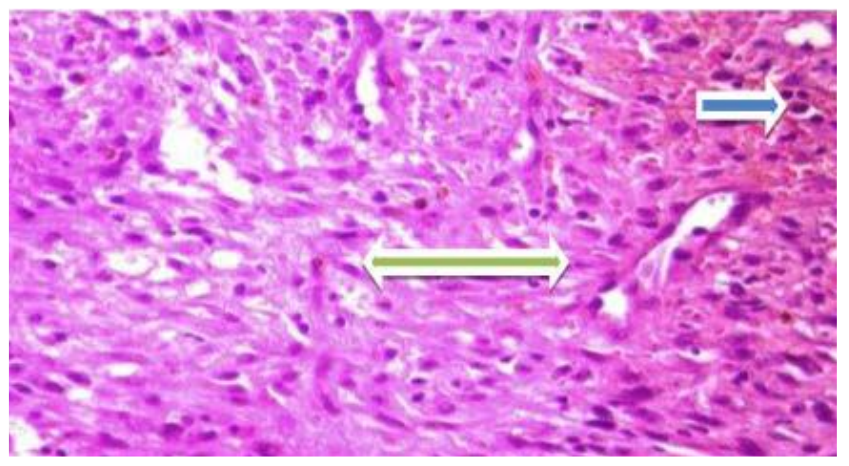

Fig. 5: Histopathologic section of wound related totreatment group on day $7^{\text {th }}$ post-wounding, show's granulation tissue replaced most of the incisional area (green arrow), with congested blood vessels, neutrophils and mononuclear cells infiltration (blue arrow) (H\&E; $\mathrm{X} 40)$.

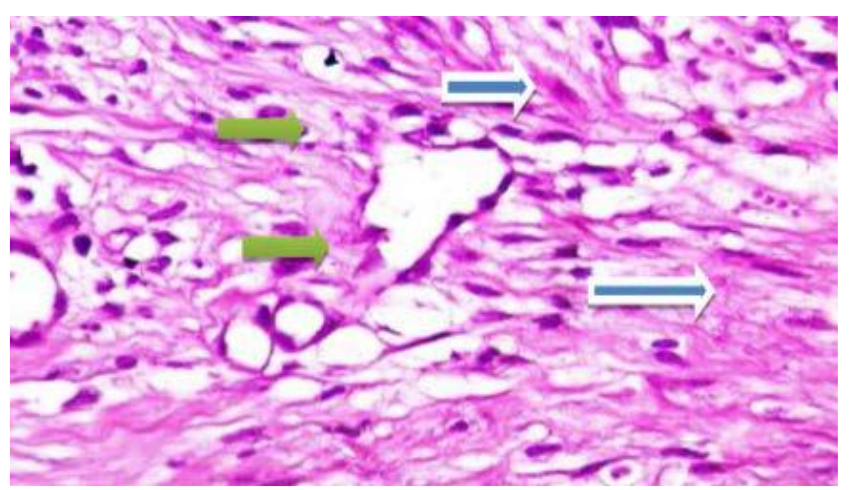

Fig. 6: Histopathologic section of wound related to control group, 14 days post-wounding, show's immature granulation tissues (blue arrows) with mononuclear cells infiltration (green arrows) (H\&E; X40).

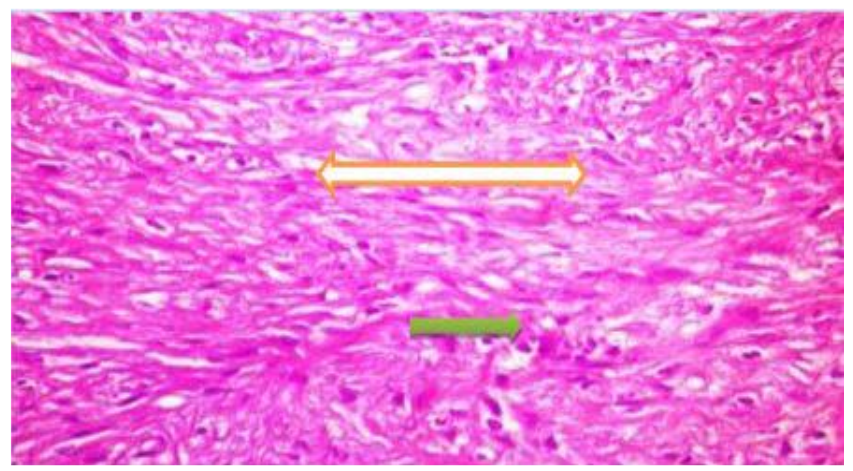

Fig. 7: Histopathologic section of wound related to treatment group, 14 day post-wounding, show's, mature granulation tissue in the incisional area (white arrow), with mononuclear cells infiltration (green arrow) (H\&E; X40).

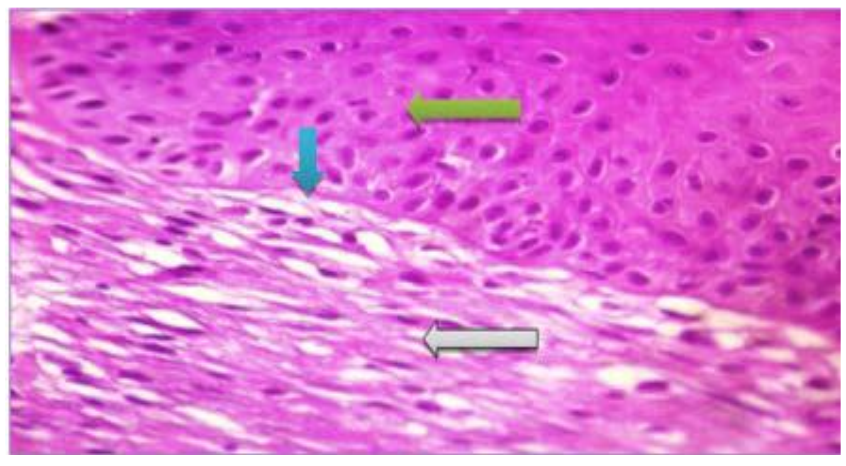

Fig. 8: Histopathologic section of wound related tocontrol group, 21days post-wounding, show's thick epidermal layer (green arrow) over the granulation tissue (grey arrow) with short rete ridge (blue arrow) (H\&E; X40).

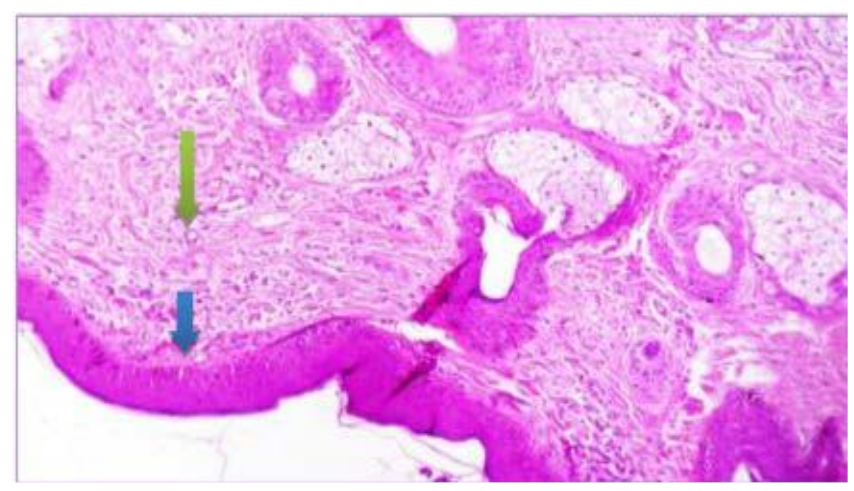

Fig. 9: Histopathologic section of wound related to treatment group, 21days post-wounding shows normal epidermal area (blue arrow) covers mature granulation tissue (green arrow). (H\&E; X20).

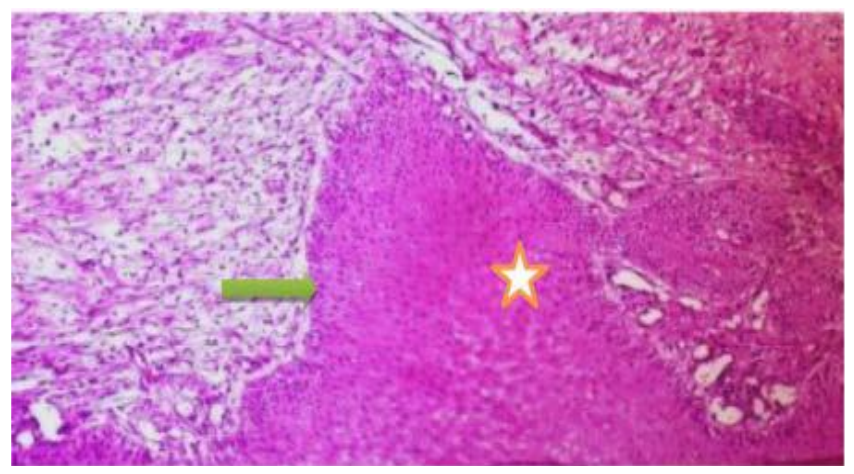

Fig. 10: Histopathologic section of wound related to control group, 28 days post-wounding, show's the presence of epidermis layer (star) cover the mature granulation tissue (green arrow). (H\&E; X40). 


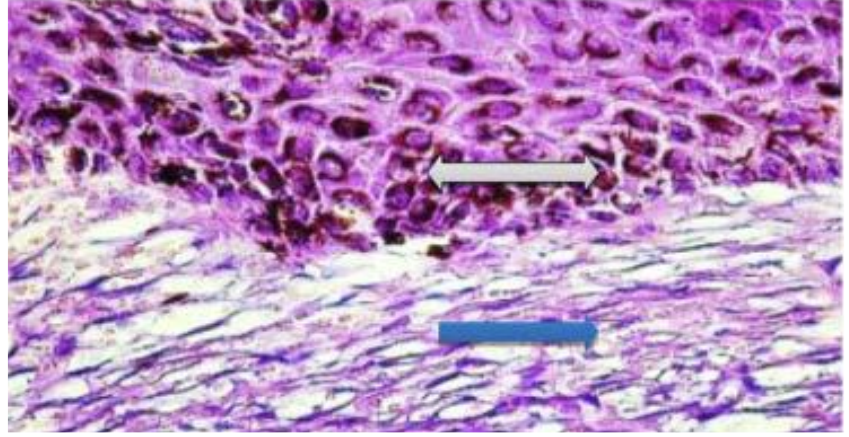

Fig. 11: Histopathologic section of wound related to treatment group, 28 days post-wounding, show's melanin pigment in the basal layer of epidermis (grey arrow) cover the granulation tissue (blue arrow) (H\&E; X40).

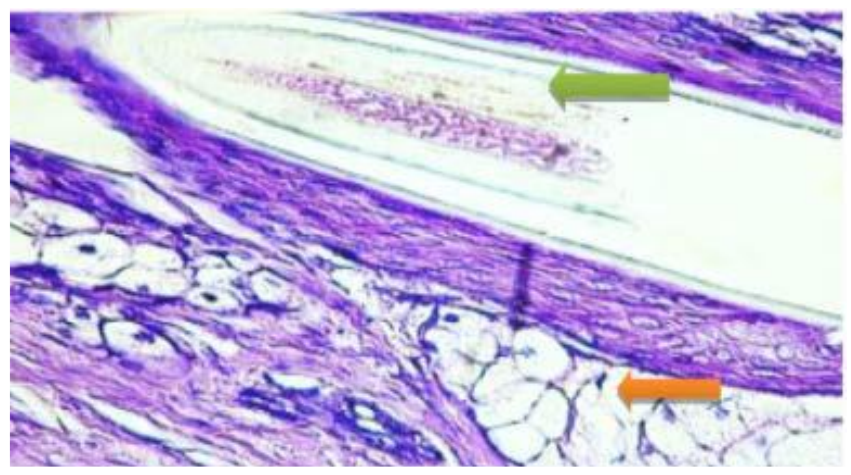

Fig. 12: Histopathologic section of wound related to treatment group, 35 day post-wounding, show's the reformation of hair follicles (green arrow) and sebaceous glands (orang arrow) in the incisional site (H\&E; X40).

\section{Discussion}

Amid a follow-up for 35 days, we did not record any secondary complications of the wounds in both groups; this may ascribe to highly strike antiseptic and appropriated post-operative cares. These findings were in accordance with (14) in dog. Interestingly (19), watched diseases in two injuries out of 32 when utilized PRP for treatment of cutaneous injuries in horse. These distinctions in the consequences of the investigations might be identified with the quantity of creatures utilized as a part of each examination. The imperative parameters were hoisted in the primary week post-injuring in the two gatherings yet with no altogether contrasts $\mathrm{P}>0.05$. These augmentations in parameters level might be ascribed to intense aggravation evocated from injuring. At that point the qualities held step by step to its typical levels. These findings were in agreement with previous study (30). The morphometric assessment of wounds in the present examination, demonstrated that the advance of epithelialization was speedier in twisted treated with PRP contrasted and control wounds amid four week contemplate. It has been exhibited that PRP provoked cutaneous injury repair by means of separation into different skin cell writes including; keratinocytes, endothelial cells, pericytes and monocytes, (31). (32), Recommended that PRP engrafted in cutaneous injury quicken re-epithelialization, through their capacity to animate the keratinocytes to separate into different epithelial cell composes, for example, skin epithelial cells, after foundational organization in vivo. Re-epithelialization of wounds starts inside hours after damage. Epidermal cells from skin members, for example, hair follicles rapidly evacuate thickened blood and harmed stroma from the injury space (33). The statement of integrin receptors on epidermal cells enables them to associate with an assortment of Extracellular-Matrix (ECM) proteins (e.g., fibronectin and vitronectin) that are blended with stromal type I collagen at the edge of the injury and joined with the fibrin coagulation in the injury space. As reepithelialization follows, cellar layer proteins return in an extremely requested succession from the edge of the injury internal. Epidermal cells return to their ordinary phenotype, by and by immovably connecting to the restored storm cellar layer and hidden dermis (34). The after effects of current examination demonstrated that injury compression percent was more prominent in treatment twisted contrasted with the control wound. (35). Demonstrated that in secondexpectation wound recuperating, conclusion is accomplished by constriction and epithelialization. A more prominent commitment of wound compression quickens recuperating in light of the fact that constriction happens quicker than epithelialization. Wound withdrawal is characterized as the centripetal development of the first twisted edges. This procedure happens because of compression of myofibroblasts in granulation tissue. Myofibroblasts are basic to wound withdrawal and mending. They separate from fibroblast and are portrayed by nearness of stress strands containing $\alpha$-actin isoform that is communicated in smooth muscle. It has been affirmed that PRP communicated Transforming Growth Factor (TGF) which initiate fibroblasts and separate into my fibroblast and increment the quantity of these cells to advance injury constriction (36).

Wound contraction involves a complex and superbly orchestrated interaction of cells, ECM and cytokines. During the second week of healing, fibroblasts assume a my fibroblast phenotype characterized by large bundles of actin-containing microfilaments disposed along the cytoplasmic face of the plasma membrane of the cells and by cell-cell and cell-matrix linkages (37), the appearance of the myofibroblasts corresponds to the commencement of connective-tissue compaction and the contraction of the wound. The contraction probably requires stimulation by 
TGF and Platelet-Derived Growth Factor (PDGF) attachment of fibroblasts to the collagen matrix through integrin receptors and cross-links between individual bundles of collagen. Collagen rebuilding amid the progress from granulation tissueto scar is dependent on continued synthesis and catabolism of collagen at a low rate. The debasement of collagen in the injury is controlled by a few proteolytic catalysts named network metalloproteinase, which are discharged by macrophages (38). Histopathological areas, uncovers discharge and provocative cells amid the initial seven days notwithstanding the arrangement of granulation tissue. These perceptions were recorded by (39), in dogs in which the new stroma, often called granulation tissue, begins to invade the wound space approximately four days after injury. Numerous new capillaries endow the new stroma with its granular appearance. Macrophages, fibroblasts and blood vessels move into the wound space at the same time. The macrophages provide a continuing source of growth factors necessary to stimulate fibroplasia and angiogenesis; the fibroblasts produce the new ECM necessary to support cell in growth; and blood vessels carry oxygen and nutrients necessary to sustain cell metabolism. (40), indicated that growth factors, especially PDGF and TGF in concert with the ECM molecules, presumably stimulate fibroblasts of the tissue around the wound to proliferate and produce collagen fibers which resemble a bridge connecting the ends of the wound in addition, the fibroblasts are responsible for the synthesis, deposition and remodeling of the extracellular matrix. The formation of new blood vessels is necessary to sustain the newly formed granulation tissue. Angiogenesis is a complex process that relies on extracellular matrix in the wound bed as well as migration and mitogenic stimulation of endothelial cells (32). In the present study, the re-epithelialization of epidermis in PRP treatment group started at the second week post-wounding while it appeared in fourth week in control group and this continuation was supported by a study of (41). On another hand, (42) stated that Re-epithelialization of the wound can be conceptually viewed as the result of three overlapping keratinocyte functions: migration, proliferation and differentiation. The sequence of events by which keratinocytes accomplish the task of re-epithelialization is generally believed to begin with dissolution of cell-cell and cell-substratum contacts. This is followed by the polarization and initiation of migration in basal and a subset of supra-basilar keratinocytes over the provisional wound matrix.

Well differentiated keratinocytes of epidermis with scar formation in the dermis were observed in treatment group at day 28. While, in randomized prospective study by Dyson (43), showed that scar formation at day 35th post-wounding and indicated that the epidermis regenerated progressively from the surrounding wound margins. The neoepidermis showed a complete spectrum of changes. Near the wound margin, the differentiation of the neoepidermis and regeneration of the dermo-epidermal junction were more advanced than toward the wound center, where the proliferative index was significantly increased. Also Bennett and Schultz, (16), referred that the wound becomes covered with epithelial tissue within two weeks, minimal collagen will be deposited and no scar will form. Generally, if a wound takes longer than three to four weeks to become covered, a scar will form.

\section{Conclusions}

The present study concluded that the procedure of local implantation of PRP is easy and safe. This study demonstrates the beneficial effect of PRP in cutaneous wound healing via fast epithelialization and effective wound contraction.

\section{Acknowledgments}

I wish to express my deep appreciation to the staff of surgery department, College of Veterinary Medicine, University of Baghdad.

\section{References}

1. Diegelmann RF, Evans MC Wound healing: an overview of acute, fibrotic and delayed healing. Front Biosci. 2004;9:283-289.

2. Kairuz EZ, Upton RA. Dawson A, Malda J. Hyperbaric oxygen stimulates epidermal reconstruction in skin equivalents. Wound Repair Regen. 2007;15:266-274.

3. Frechette JP, Martineau I, Gagnon G. Platelet-rich Plasmas: Growth Factor Content and Roles in Wound Healing. J Dent Res. 2005;84(5):434-439.

4. Kairuz E, Upton Z, Dawson RA, Malda J. Hyperbaric oxygen stimulates epidermal reconstruction in skin equivalents. Wound Repair Regen. 2007;15:266-274.

5. Priya KS, Arumugam G, Rathinam B, Wells A, Babu M. Local injection of insulin-zinc stimulates DNA synthesis in skin donor site wound. Wound Repair Regen. 2007;15: 258-265.

6. Ferguson M, Byrnes C, Sun L. Wound healing enhancement: Electroportionto address a classic problem of military medicine. World J Surg. 2005;29:S55-S59.

7. Mann A, Breuhahn K, Schirmacher P. Keratinocyte-derived granulocytemacrophage colony stimulating factor accelerates wound healing: stimulation of keratinocyte proliferation, granulation tissue formation, and vascularization. J Invest Dermatol. 2001;117:13821390.

8. Goren I, Müller E, Schiefelbein D, Systemic anti-TNFalpha treatment restores diabetes-impaired skin repair in ob/ob mice by inactivation of macrophages. J Invest Dermatol. 2007;127:2259-2267.

9. Monteiro SO, Lepage OM, Theoret CL. Effects of platelet-rich plasma on the repair of wounds on the distal aspect of the forelimb in horses. Am J Vet Res. 2009;70(2):277-282.

10. Steel CM, Robertson ID, Thomas J. Effect of topical rh-TGF- $\beta 1$ on secondintention wound healing in horses. Aust Vet J. 1999;77:734737.

11. Wu L, Xia YP, Roth SI. Transforming growth factor-beta1 fails to stimulatewound healing and impairs its signal transduction in an aged ischemic ulcer model: importance of oxygen and age. Am J Pathol. 1999;154:301-309. 
12. Badylak SF, Xenogeneic extracellular matrix as a scaffold for tissue reconstruction. Transpl Immunol. 2004;12:367-377.

13. Lee SH, Lee JH, Cho KH. Effects of human adipose-derived stem cells on cutaneous wound healing in nude mice. Ann Dermatol. 1999;23(2):150-151.

14. Azari O, Babae HI, Molaei MM, Nematollahi-Mahani SN, Layasi ST. The use of Wharton's jelly-derived mesenchymal stem cells to accelerate second-intention Cutaneous wound healing in goat. Iran $\mathbf{J}$ Vet Surg. 2008;3(8):15-27.

15. Kazakos K, Lyras DN, Verettas K. The use of autologous PRP gel as an aid in the management of acute trauma wounds. Injury.2009;40:801-805.

16. Bennett NT, Schultz GS. Growth factors and wound healing: Part II. Role in normal and chronic wound healing. Amer J Surg. 1993;166:74-8118.

17. Wieman TJ. Clinical efficacy of becaplermin (rhPDGF-BB) gel. Becaplermin GelStudies Group. Am J Surg. 1998;176:74S-79S.

18. Chen C, Schultz GS, Bloch M. Molecular and mechanistic validation of delayed healing rat wounds as a model for human chronic wounds. Wound Rep Regen.1999;7(6):486-494.

19. Trengove NJ, Bielefeldt-Ohmann H, Stacey MC. Mitogenic activity and cytocinelevels in non-healing and healing chronic leg ulcers. Wound Rep Regen. 2000;8(1):13-25.

20. Kirchne RLM, Meerbaum SO, Gruber BS. Effects of vascular endothelial growth factor on wound closure rates in the genetically diabetic mouse model. Wound Rep Reg. 2003;11:127-131.

21. Galiano RD, Tepper OM, Pelo CR. Topical vascular endothelial growth factoraccelerates diabetic wound healing through increased angiogenesis and by mobilizingand recruiting bone marrow-derived cells. Am J Pathol. 2004;164:1935-1947.

22. Lee HW, Reddy MS, Geurs N. Efficacy of platelet-rich plasma on wound healing in rabbits. J Periodontol. 2008;79(4):691-696.

23. Kim JH, Park C, Park HM. Curative effect of autologous platelet-rich plasma on a large cutaneous lesion in a dog. Vet Dermatol. 2009;20(2):123-126.

24. Woessner JF. The determination of hydroxyproline in tissue and protein samples containing small proportions of this amino acid. Arch Biochem Biophys. 1961;91:440-447.

25. Kimura A, Ogata H, Yazawa M, Watanabe N, Mori T, Nakajima T. The effects of platelet-rich plasma on cutaneous incisional wound healing in rats. J Dermatol Sci. 2005;40:205-208.

26. Kevy S, Jacobson M. Comparison of methods for point of care preparation of autologous platelet gel. J Extra-Corp Technol. 2004;36:28-35.

27. Bohling MW, Henderson RA, Swaim SF, Kincaid SA, Wright JC. Cutaneous wound healing in cat: macroscopic description and comparison with cutaneous wound healing in dogs. Vet Surg. 2004;33:579-587.

28. Luna LG. Hematoxylin and Eosin Stain: Histological Staining Method of the Armed Forces Institutes of Pathology. 3rd Edn. McGraw-Hill, Book Co., New York.1968; pp:40-41.

29. SAS. Statistical Analysis System, User's Guide.Statistical.Version $7^{\text {th }}$ ed. SAS.Inst. Inc. Cary.N.C. USA. 2004.

30. Crovetti GG. Issi M. Platelet gel for healing chronic wounds. Transfus Apher Sci. 2004;30:145-151.

31. Anitua ME, Sanchez A, Nurden P, Nurden G, Andia I. New insights and novel applications for platelet-rich fibrin therapies. Trends Biotechnol. 2006;24(5):227-234.

32. Wu Y, Chen L, Scott PG. Mesenchymal stem cells enhance wound healing through differentiation and angiogenesis. Stem Cells. 2007;25:2648-2654.

33. Kirsner RS, Eaglstein WH. The wound healing process. Dermatol Clin. 1993;11:629-640.

34. Leitner GC, Gruber R, Neumülle Jr, Körmöczi G, Buchta C. Platelet content and growth factor release in platelet rich-plasma: A comparison of four different systems. Vox Sang. 2006;91(2):135-139.

35. Swaim SF, Hinkle SH, Bradley DM. Wound contraction: Basic and clinical factors. Compendium. 2001;23:20-24.

36. Beckert SS, Haack H, Hierlemann F, Farrahi P, Mayer A. Coerper S. Stimulation of steroid-suppressed cutaneous healing by repeated topical application of IGF-I: Different mechanisms of action based upon the mode of IGFI delivery. J Surg Res. 2007;139: 217-221.

37. Masur SK, Dewal HS, Dinh TT. Myofibroblasts differentiates from fibroblasts when plated at low density. P. Natl Acad Sci.1996;93:4219-4223.

38. Liu Y, Kalen A, Risto O. Fibroblast proliferation due to exposure to a platelet concentrate in vitro is $\mathrm{pH}$ dependent. Wound Repair Regen. 2002;10:336-340.

39. Mehrjerdi HK, Sardari K, Emami M R, Movassaghi AR, Goli AA, Lotfi A. Efficacy of Autologous Platelet-Rich Plasma (PRP) Activated By Thromboplastin-D on the Repair and Regeneration of Wounds in Dogs. Iranian J Vet Surg. 2008;3:4(9):19-30

40. Werner S, Grose R. Regulation of wound healing by growth factors and cytokines. Physiol Rev. 2003;83:835-870.

41. Knighton DR, Ciresi K, Fiegel VD. Stimulation of repair in chronic non-healing cutaneous ulcers: A prospective randomized blinded trial using platelet-derived wound healing formula. Surg Gynecol Obstet. 1990;170:56-60.

42. Wynn TA. Common and unique mechanisms regulate fibrosis in various fibro-proliferative diseases. J Clin Invest. 2007;117:524-529.

43. Dyson M. Advances in wound healing physiology: The comparative perspective. Vet Dermatol. 1997;8(4):227-233. 